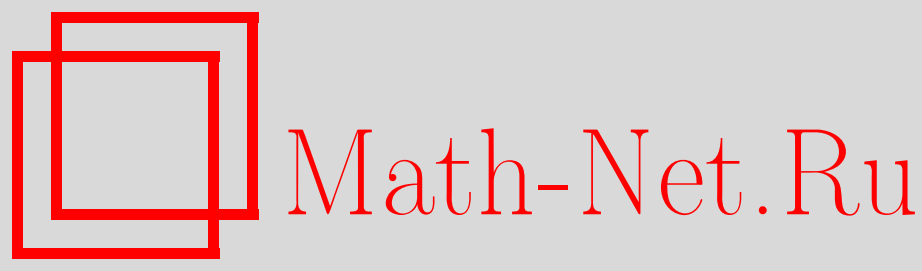

С. С. Герштейн, А. А. Логунов, М. А. Мествиришвили, Об отсутствии гравитационного излучения нестатического сферически-симметричного тела в релятивистской теории гравитации, ТМФ, 2009, том 161, номер 1, 109 114

DOI: https://doi.org/10.4213/tmf6423

Использование Общероссийского математического портала Math-Net.Ru подразумевает, что вы прочитали и согласны с пользовательским соглашением http://www.mathnet.ru/rus/agreement

Параметры загрузки:

IP : 3.93.64.190

26 апреля 2023 г., 16:28:55

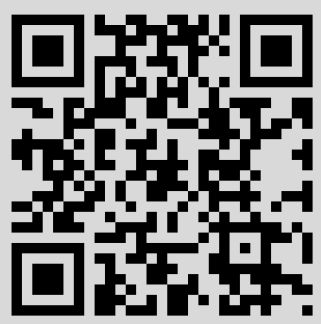




\section{ОБ ОТСУТСТВИИ ГРАВИТАЦИОННОГО ИЗЛУЧЕНИЯ НЕСТАТИЧЕСКОГО СФЕРИЧЕСКИ-СИММЕТРИЧНОГО ТЕЛА В РЕЛЯТИВИСТСКОЙ ТЕОРИИ ГРАВИТАЦИИ}

В рамках релятивистской теории гравитации установлено отсутствие гравитационного излучения нестатического сферически-симметричного источника.

Ключевые слова: масса покоя гравитона, гравитационные волны.

В общей теории относительности (ОТО) Биркгоффом [1] было установлено, что внешнее гравитационное поле нестатического сферически-симметричного тела является статическим. Это означает, что радиальные пульсации, происходящие внутри тела, не приводят к гравитационному излучению. В работе [2] в рамках релятивистской теории гравитации (РТГ), в которой гравитационное поле рассматривается как физическое поле в пространстве Минковского, было установлено, что у нестатического сферически-симметричного тела, описываемого интервалом

$$
d s^{2}=U(t, r) d t^{2}-V(t, r) d r^{2}-W^{2}(t, r)\left(d \Theta^{2}+\sin ^{2} \Theta d \Phi^{2}\right),
$$

внешнее гравитационное поле также является статическим. Однако интервал (1) не является общим для подобного нестатического сферически-симметричного тела. Общий вид интервала для уединенного нестатического сферически-симметричного тела, окруженного пустым пространством, свободным от вещества, имеет вид

$$
\begin{aligned}
d s^{2}= & U(t, r) d t^{2}-V(t, r) d r^{2}+2 E(t, r) d r d t- \\
& -W^{2}(t, r)\left(d \Theta^{2}+\sin ^{2} \Theta d \Phi^{2}\right)
\end{aligned}
$$

Под веществом мы подразумеваем все формы материи, кроме гравитационного поля. Ввиду сложности системы нелинейных уравнений РТГ нам не удалось доказать статичность внешнего гравитационного поля для источника, описываемого интервалом (2), однако для принципиального ответа на вопрос, излучает ли такой источник гравитационные волны, есть более простой путь. Так как излучаемая источником

* Институт физики высоких энергий, г. Протвино, Московская обл., Россия. E-mail: Semen.Gershtein@ihep.ru, Anatoly.Logunov@ihep.ru 
энергия нигде не накапливается и не исчезает, достаточно определить плотность гравитационного потока в асимптотической области вдали от источника, где гравитационное поле заведомо слабое. Об этом подробнее ниже.

В РТГ физическое тензорное гравитационное поле $\Phi^{\mu \nu}$ развивается в пространстве Минковского. Источником поля является сохраняющийся тензор энергии-импульса вещества и гравитационного поля вместе взятых. На этой основе возникает эффективное риманово пространство полевого происхождения с метрическим тензором $g_{\mu \nu}$, который связан с полем $\Phi^{\mu \nu}$ соотношением $\widetilde{g}^{\mu \nu}=\widetilde{\gamma}^{\mu \nu}+\widetilde{\Phi}^{\mu \nu}$, где $\widetilde{g}^{\mu \nu}=\sqrt{-g} g^{\mu \nu}, \widetilde{\gamma}^{\mu \nu}=\sqrt{-\gamma} \gamma^{\mu \nu}, \widetilde{\Phi}^{\mu \nu}=\sqrt{-\gamma} \Phi^{\mu \nu}$. Система уравнений гравитационного поля в РТГ принимает общековариантный вид

$$
R_{\mu \nu}=8 \pi\left(T_{\mu \nu}-\frac{1}{2} g_{\mu \nu} T\right)+\frac{m^{2}}{2}\left(g_{\mu \nu}-\gamma_{\mu \nu}\right),
$$

к ней добавляется общековариантная система уравнений для вещества

$$
\nabla_{\nu} T^{\mu \nu}=0
$$

Физическое решение этих уравнений должно удовлетворять условию причинности, о котором будет сказано ниже. Уравнения (3), (4) вместе с уравнением состояния вещества образуют полную систему, если вещество описывается физическими величинами: плотностью $\rho$, давлением $p$ и скоростью $\vec{v}$. Из уравнений $(3),(4)$ следует уравнение

$$
D_{\nu} \widetilde{g}^{\mu \nu}=0
$$

где $D_{\nu}$ - ковариантная производная в пространстве Минковского. Для данного вещества систему уравнений (5) можно использовать вместо уравнений (4). Уравнение (5) общековариантны и в инерциальной системе в галилеевых координатах принимают вид гармонических условий, которые часто используются в ОТО в качестве дополнительных нековариантных координатных условий, $\partial_{\nu} \widetilde{g}^{\mu \nu}=0$. В ОТО для нахождения физического решения уравнений

$$
R_{\mu \nu}-\frac{1}{2} g_{\mu \nu} R=8 \pi T_{\mu \nu}
$$

обычно налагают дополнительные требования: условие причинности Гильберта и условие положительной определенности $T_{\mu \nu} K^{\mu} K^{\nu} \geqslant 0$, где $K^{\nu}$ - произвольный времениподобный вектор.

Шоке-Бруа установила существование и единственность решения внешней локальной задачи Коши в ОТО. Существование и единственность внешнего решения сохраняется и для системы уравнений (3), (5). В РТГ источник не задан, он определяется из уравнений гравитационного поля (3), уравнений вещества (4) и условия причинности. Суть последнего состоит в том, что, поскольку физическое гравитационное поле $\Phi^{\mu \nu}$ развивается в пространстве Минковского, в каждой точке пространства конус причинности возникшего в РТГ эффективного риманова пространства должен находиться внутри конуса причинности пространства Минковского. Условие причинности налагает требование на решение уравнений (3), (5). 
В РТГ, как мы видим, с необходимостъю возникла масса покоя гравитона. Обычно введение массы покоя гравитона в теорию гравитации приводило к появлению нефизических "духовых" состояний. Так, в работах [3], [4] было установлено, что в линеаризованной теории гравитации введение массы покоя гравитона в поле со спинами 2 и 0 приводит при попытке объяснения гравитационных эффектов в Солнечной системе к появлению нефизических "духовых" состояний, возникших из-за наличия гравитона со спином 0. "Духовые" состояния возникают и в гравитационном излучении от нестатического сферически-симметричного тела. Однако авторы работ [3], [4] в своих исследованиях не рассматривали гравитационное поле как тензорное физическое поле в пространстве Минковского, создающее своим действием эффективное риманово пространство. Но именно это приводит к появлению дополнительно к конусу причинности пространства Минковского еще и конуса причинности эффективного риманова пространства. Наличие двух конусов причинности с необходимостью требует введения условия причинности РТГ.

Из лоренц-инвариантности теории и условия причинности в пространстве Минковского с необходимостью вытекает требование, чтобы в каждой точке пространства конус причинности эффективного риманова пространства находился внутри конуса причинности пространства Минковского. Для того чтобы это требование выполнялось, необходимо и достаточно, чтобы при наличии неравенства $g_{\mu \nu} p^{\mu} p^{\nu} \geqslant 0$ было бы справедливо также неравенство $\gamma_{\mu \nu} p^{\mu} p^{\nu}>0$. Из этих неравенств следует, что для нестатического сферически-симметричного тела, описываемого интервалом (2), имеет место неравенство

$$
V-U>2|E|
$$

В случае сферически-симметричного статического поля $E \equiv 0$. Для слабого гравитационного поля метрический тензор эффективного риманова пространства связан с полем $\Phi_{\mu \nu}$ равенством

$$
g_{\mu \nu}=\gamma_{\mu \nu}-\Phi_{\mu \nu}+\frac{1}{2} \gamma_{\mu \nu} \Phi, \quad \Phi=\Phi_{\mu \nu} \gamma^{\mu \nu}
$$

Отсюда следует, что

$$
U=1-\Phi_{00}+\frac{1}{2} \Phi, \quad V=1+\Phi_{11}+\frac{1}{2} \Phi
$$

Подставляя эти выражения в неравенство (7), получим

$$
\Phi_{11}+\Phi_{00}>2|E|,
$$

где согласно (8) $E=-\Phi_{01}$. Этим неравенством мы будем пользоваться при нахождении физического решения гравитационных уравнений.

$B$ асимптотической области по радиальной переменной $r$ вдали от источника уравнения РТГ для слабого гравитащионного поля принимают вид [5]

$$
\begin{gathered}
\gamma^{\mu \nu} D_{\mu} D_{\nu} \Phi^{\alpha \beta}+m^{2} \Phi^{\alpha \beta}=0 \\
D_{\nu} \Phi^{\mu \nu}=0
\end{gathered}
$$


Метрический тензор пространства Минковского в сферических координатах записывается как $\gamma_{\mu \nu}=\left(1,-1,-r^{2},-r^{2} \sin ^{2} \Theta\right)$. Уравнения (11) для компонент поля в сферических координатах пространства Минковского в соответствии с интервалом (2) можно записать в форме

$$
\begin{array}{r}
\frac{\partial^{2} \Phi_{0}^{0}}{\partial t^{2}}-\frac{\partial^{2} \Phi_{0}^{0}}{\partial r^{2}}-\frac{2}{r} \frac{\partial \Phi_{0}^{0}}{\partial r}+m^{2} \Phi_{0}^{0}=0, \\
\frac{\partial^{2} \Phi_{1}^{0}}{\partial t^{2}}-\frac{\partial^{2} \Phi_{1}^{0}}{\partial r^{2}}-\frac{2}{r} \frac{\partial \Phi_{1}^{0}}{\partial r}+\frac{2}{r^{2}} \Phi_{1}^{0}+m^{2} \Phi_{1}^{0}=0 \\
\frac{\partial^{2} \Phi_{1}^{1}}{\partial t^{2}}-\frac{\partial^{2} \Phi_{1}^{1}}{\partial r^{2}}-\frac{2}{r} \frac{\partial \Phi_{1}^{1}}{\partial r}+\frac{4}{r^{2}} \Phi_{1}^{1}-\frac{4}{r^{2}} \Phi_{2}^{2}+m^{2} \Phi_{1}^{1}=0 \\
\frac{\partial^{2} \Phi_{2}^{2}}{\partial t^{2}}-\frac{\partial^{2} \Phi_{2}^{2}}{\partial r^{2}}-\frac{2}{r} \frac{\partial \Phi_{2}^{2}}{\partial r}+\frac{2}{r^{2}} \Phi_{2}^{2}-\frac{2}{r^{2}} \Phi_{1}^{1}+m^{2} \Phi_{2}^{2}=0
\end{array}
$$

Уравнения (12) в тех же координатах в соответствии с интервалом (2) имеют вид

$$
\begin{array}{r}
\frac{\partial \Phi_{0}^{0}}{\partial t}+\frac{\partial \Phi_{0}^{1}}{\partial r}+\frac{2}{r} \Phi_{0}^{1}=0 \\
\frac{\partial \Phi_{1}^{0}}{\partial t}+\frac{\partial \Phi_{1}^{1}}{\partial r}-\frac{1}{r} \Phi_{2}^{2}-\frac{1}{r} \Phi_{3}^{3}+\frac{2}{r} \Phi_{1}^{1}=0 .
\end{array}
$$

В соответствии с интервалом (2) имеет место также равенство

$$
\Phi_{2}^{2}=\Phi_{3}^{3}
$$

Подъем и опускание индексов у полевой переменной $\Phi^{\mu \nu}$ осуществляется с помощью метрического тензора пространства Минковского $\gamma_{\mu \nu}$. В асимптотической области по радиальной переменной $r$ из уравнения (13) находим для волны единственное решение вида

$$
\Phi_{00}=\frac{r_{\mathrm{g}}}{r} e^{-m r}+a_{00}(\omega) \frac{\cos \omega(t-q r)}{r}, \quad q=\left(1-\frac{m^{2}}{\omega^{2}}\right)^{1 / 2} .
$$

В этом решении первый член учитывает статическое поле источника, а второй член представляет расходящуюся от источника сферическую волну. Для простоты вычислений мы взяли одну волну с частотой $\omega$.

Для решений типа (20) в уравнениях (14), (15), (17) и (18) можно пренебречь членами, убывающими с ростом $r$ как $1 / r^{2}$. После такого упрощения уравнений из (14) и (15) мы находим

$$
\begin{aligned}
& \Phi_{01}=a_{01}(\omega) \frac{\cos \omega(t-q r)}{r} \\
& \Phi_{11}=\frac{r_{\mathrm{g}}}{r} e^{-m r}+a_{11}(\omega) \frac{\cos \omega(t-q r)}{r} .
\end{aligned}
$$

В том же приближении из уравнений (17) и (18) получим в асимптотической по $r$ области соотношения

$$
\Phi_{00}=-q \Phi_{01}+\frac{r_{\mathrm{g}}}{r} e^{-m r}, \quad \Phi_{11}=-\frac{\Phi_{01}}{q}+\frac{r_{\mathrm{g}}}{r} e^{-m r} .
$$


При получении формул (21), (22) мы пренебрегли ввиду малости $m$ членами вида $\left(m r_{\mathrm{g}} / r\right) e^{-m r}$.

С учетом равенства (22) неравенство (10) принимает вид

$$
-\frac{q^{2}+1}{q} r \Phi_{01}>2 r\left|\Phi_{01}\right|-2 r_{\mathrm{g}} e^{-m r}
$$

При фиксированном значении разности $t-q r$ левая часть последнего неравенства, а также первый член в правой части не зависят от $r$, тогда как второй член при $r \rightarrow \infty$ обращается в нуль, и, поскольку функция $\Phi_{01}$ знакопеременна, для выполнения неравенства необходимо положить

$$
a_{01} \equiv 0
$$

Следовательно согласно равенствам (22) мы имеем

$$
a_{00} \equiv 0, \quad a_{11} \equiv 0 .
$$

На основании (24) и (25) можно утверждать, что в расходящейся физической волне отсутствуют продольно-продольные компоненты. Решения в форме продольно-продольных волн не удовлетворяют принципу причинности РТГ.

В РТГ в квадратичном приближении плотность потока гравитационной энергии, в отличие от ОТО, определяется согласно [5], [6] тензорной величиной

$$
t_{g}^{\epsilon \lambda}=\frac{1}{32 \pi} \gamma^{\epsilon \alpha} \gamma^{\lambda \beta}\left(D_{\alpha} \Phi_{\nu}^{\tau} \cdot D_{\beta} \Phi_{\tau}^{\nu}-\frac{1}{2} \partial_{\alpha} \Phi \cdot \partial_{\beta} \Phi\right)
$$

Отсюда плотность потока равна

$$
t_{g}^{01}=-\frac{1}{32 \pi}\left(D_{0} \Phi_{\nu}^{\tau} D_{1} \Phi_{\tau}^{\nu}-\frac{1}{2} \partial_{0} \Phi \cdot \partial_{1} \Phi\right) .
$$

Для гравитационного поля, описываемого интервалом (2) с учетом равенств (24), (25), после усреднения по времени на основании (27) находим поток гравитационной энергии в единицу времени в элемент телесного угла:

$$
\frac{d I}{d \Omega}=\frac{1}{128 \pi} q \omega^{2}\left(a_{2}^{2}-a_{3}^{3}\right)^{2}
$$

Но так как для сферически-симметричного интервала (2) имеет место равенство (19), эта величина в асимптотической по $r$ области будет равна нулю:

$$
\frac{d I}{d \Omega}=0
$$

Поскольку поток энергии, если он существует, не может обратиться в нуль, на основании (29) можно утверждать, что нестатическое сферически-симметричное тело в РТГ также не излучает гравитационные волны. Если теперь воспользоваться равенствами $(19),(24),(25)$ и учесть их в уравнении (18), то мы придем к выводу, что $a_{2}^{2}=a_{3}^{3} \equiv 0$. Это означает, что все компоненты расходящейся волны равны нулю.

5 Теоретическая и математическая физика, т. 161, № 1, 2009 г. 


\section{Список литературы}

[1] G. D. Birkhoff, R. E. Langer, Relativity and Modern Physics, Harward Univ. Press, Cambridge, 1923.

[2] A. A. Logunov, M. A. Mestvirishvili, Phys. Part. Nucl., 40:1 (2009), 67-70.

[3] В. И. Захаров, Писъма в ЖЭТФ, 12:9 (1970), 447-449.

[4] H. van Dam, M. Veltman, Nucl. Phys. B, 22:2 (1970), 397-411.

[5] А. А. Логунов, Релятивистская теория гравитации, Наука, М., 2006.

[6] Ю. М. Лоскутов, ТМФ, 107:2 (1996), 329-343. 